realization of probabilities. A variety of verbal methods of description can be used for this, from which Dr. Scott Blair has chosen some.

In the unique pattern of world lines appropriate to a completely deterministic world, it is not clear how an analysis would reveal the rules of causality (the physical laws). Since every set of events would be in some way unique, any selection of apparently recurring patterns would be arbitrary.

Such sets of recurring patterns would be the only way of investigating possible causal relations. It would, however, still require an arbitrary judgment to say which patterns represent direct cause-effect relations and which represent the various effects of some previous cause. In a deterministic world, it would be necessary to understand all causal connexions in the entire pattern before any particular laws of causality could be singled out.

In a non-deterministic system the situation is quite different. If it is found that certain events occur invariably after the occurrence of some uncaused event, but not in its absence, then a causal connexion can immediately be inferred, for no such events can he regarded as distant effects of earlier causes.

Mr. C. O. Hines presents a completely acceptable definition of the velocity $c$ from the experimental verification of the Lorentz transformation. But he does not point out that this implies a limiting velocity $c$ also for the transfer of information if cause-effect relations are to be unidirectional in time. We are therefore led back to what Mr. Hines calls principle $P$, and so to the starting point of this discussion.

The essential fact is that no step-by-step investigation of Nature is logically possible without some indeterminacy. This statement does not conflict with the possibility of constructing deterministic mathematical theories to cover any known field, but denies their scientific utility.

Faculty of Mathematics,

University of Cambridge.

H. BONDI

Cavendish Laboratory,

University of Cambridge.

\section{Measurement of Thicknesses of Fine Particles}

A METEOD for measuring thicknesses of particles in the sub-sieve range has been devised. Measurements are made using the Vickers projection microscope. The apparatus required is an attachment to the sub-stage condenser which replaces its front lens; it consists mainly of a quartz spherical lens, which is allowed to make contact with a microscope slide carrying a dispersion of fine particles mounted dry on its uppermost surface - which is most distant from the objective lens-as shown in the diagram.

Contact is made on a strip which has been wiped free of particles, near the left-hand edge of the slide. The point of contact is focused on to the screen and is arranged to lie at the middle of the left-hand side of the screen. When the slide is traversed from right to left, particles enter the ficld and are seen to meet the lens at varying distances from the point of contact of lens and slide. These distances are proportional to the square roots of the thicknesses of the particles.
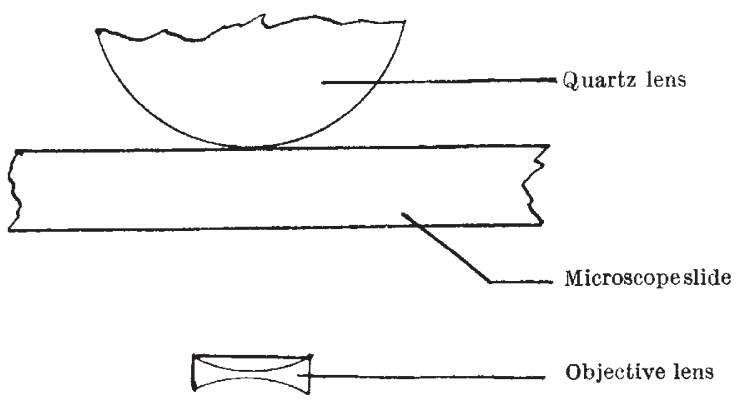

By having interchangeable spherical lenses of different diameters and the choice of suitable magnifications, lens diameter being decreased when the magnification is increased, the thicknesses of particles of diameters in the range 763 microns can be measured with the same degree of accuracy throughout, the standard error of a single measurement being 5 per cent, if the smallest thickness measured is not less than 0.4 micron.

Transmitted light is used for all measurements, and the illumination and image definition do not deteriorate even when objectives of $4 \mathrm{~mm}$. and $2 \mathrm{~mm}$. focal lengths are used. When these particular objectives are used, rectangular No. 1 microscope cover slips carry the particles.

This method of measurement is eminently suitable for the Vickers projection microscope but is not easily adapted to the normal light microscope; it could be readily adapted to the inverted microscope, measurements being made with a suitable eyepiece graticule.

The method is the subject of a patent application and more details will be reported at a later date.

W. H. M. RoBINs

36 Mayfield Avenue,

Victoria Park, Cardiff.

April 17.

\section{Scintillation Spectrometer Studies on the Decay Schemes of Silver-110, Antimony-124] and lodine-|3|}

IN order to study the decay schemes of radioisotopes, we have used two scintillation spectrometers in coincidence. The construction of the spectrometers used and the measuring technique have been described earlier ${ }^{1}$. The pulses from one of the spectrometers are analysed by a single-channel pulse analyser. Pulses corresponding to a certain energyrange are selected by the channel, and the output pulses from the analyser are fed to the sweep-trigger of an oscilloscope. The pulses from the second spectrometer are led to the vertical deflexion plates of the oscilloscope. Only pulses which are in coincidence with those selected by the pulse analyser then appear on the screen. The coincidence-rate is 10-100 pulses per minute. The screen is photographed for $\dot{a}$ few hours. A picture of the pulse-height distribution is then obtained on the photographic plate; the density at a particular point is a measure of the number of pulses with the corresponding height. The density is determined by means of a microphotometer, and, from the known density curve of the plate, one obtains the pulse-height distribution 